\title{
A New Approach on Network System Design And Implementation Based On ZigBee
}

\author{
NIU Jing ${ }^{1, a}$ \\ ${ }^{1}$ Nanyang Medical College, Nanyang 473000,China \\ a nyniujing@126.com
}

Keywords: ZigBee, Wireless connection, Protocol stack

\begin{abstract}
With the microelectronics technology, computer technology and wireless communication technology advances, promoted the rapid development of low-power multifunctional sensor, in the tiny volume can integrate information acquisition, data processing and wireless communication and other functions. This article first introduces the characteristics and current situation of the development of wireless sensor network, and the wireless sensor network technology of several common, for some need a large number of nodes, low cost, low power consumption in the field of application, much attention has been paid to use gradually mature and ZigBee wireless sensor network technology. The content of the research on the application of wireless sensor networks based on ZigBee technology has a certain reference value and practical significance, and ZigBee wireless technology in the application field of application provides basic software and hardware platform.
\end{abstract}

\section{Introduction}

Advances in microelectronic technology, computer technology and wireless communication technology to promote the rapid development of low-power multi-function sensors, it can be integrated within a small volume of information collection, a variety of functions, such as data processing and wireless communications in wireless sensor networks that is, by deploying a large number of cheap micro sensor nodes within the monitored area, a multi-hop self-organizing wireless communication via a network of systems for wireless sensor networks is the current international concern, by more highly cross-discipline Integrated hot emerging frontier areas of research, is one of the technologies of the 21st century have a huge influence [1-3]. It sensor technology, embedded computing technology, modern network and wireless communication technology together with a collection of information, send and process information, and other functions. With the development of electronic technology, wireless sensor networks into the rapid development period.

However, the wireless network technology in industrial monitoring, agriculture automation, intelligent building, bio-medical, environmental monitoring, smart home applications, such as infancy, these areas are very low data throughput requirements, and power consumption than existing standards provide low power consumption. To facilitate simple and convenient, wireless devices can be freely used by large numbers, you need a lot of wireless access points are arranged in these application areas of space, so the low price will play a key role. Sensor nodes to achieve a variety of network protocols and applications, there is some real constraints: limited energy supply, limited communication capabilities, limited computing and storage capacity.

ZigBee technology is a new short-range, low-rate wireless communication network technology, it is a range of technical solutions and Bluetooth wireless technology between markers, mainly used for short-range wireless connection, the technical standards set by IEEE 802. 15.4 Working Group to develop. ZigBee wireless technology for its short-range, low-speed, low latency, low power, low cost and other advantages to make up for the vacancy of wireless communication technology, but also to meet with sensors and self-organizing wireless sensor networks represented network requirements for wireless sensor networks do not require high bandwidth, low latency requirements but with lower power consumption. This paper studied the contents of the application of 
ZigBee-based wireless sensor network technology has some reference value and the actual meaning, and for ZigBee wireless technology to provide the relevant hardware and software applications in the application of the basic platform area.

\section{ZigBee Technology Overview}

ZigBee protocol standard as a new wireless connection technology in low-cost, low-power, low-speed network capacity, Japan has opened up new horizons. ZigBee is a short-range, low complexity, low power, low data rate, low-cost wireless communication technology, it is based on the IEEE 802.15.4 standard, communicate with each other to coordinate thousands of tiny sensors. These sensors requires very little energy, a way to relay data via radio waves from one sensor to another sensor only, high communication efficiency relative to existing wireless communication technology, ZigBee technology is the lowest power consumption and cost technologies due to the low data rate ZigBee technology and small communication range of features, but also determines the ZigBee technology suitable for carrying data traffic [4-5] .ZigBee small business technical characteristics of the decision that it is the best choice for wireless sensor networks, currently abroad, ZigBee network technology has been a certain application, which protocol stack shown in Figure 1.

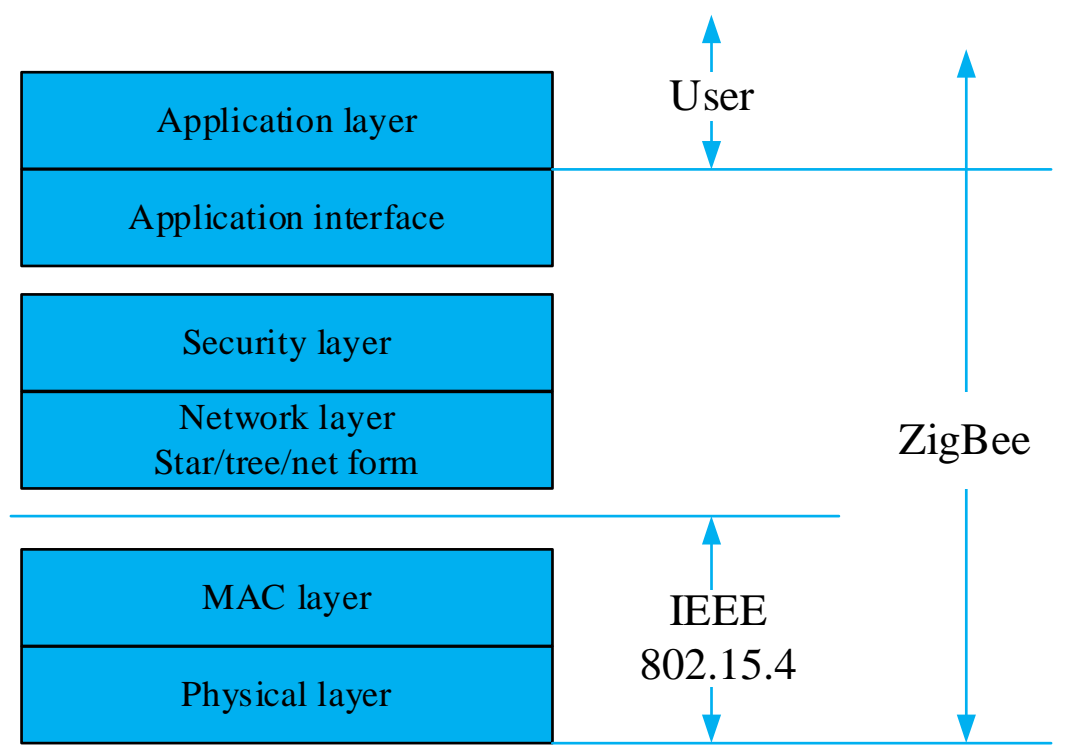

Figure 1. The ZigBee protocol stack

ZigBee protocol is relatively compact, simple, from the point of view the overall framework, can be divided into three basic levels: the physical layer / data link layer, ZigBee stack and application layer, physical layer / data link layer is located in the bottom of the application layer is located the highest level, the basic function of each layer is as follows:

Physical layer / data link layer. Physical layer and the physical transmission medium (here mainly refers to radio waves) relevant, responsible for the physical media and data bits into each other, and the data bits into each other and the upper layer) data link layer data frame. Data link layer is responsible for addressing function, determines the destination address data while sending data to send, the source address data is determined when data is received. In addition also responsible for parsing the assembly as well as of the received data frame or data packet frame.

Stack ZigBee stack layer by layer, the network layer .ZigBee and security platforms, providing connectivity applications 802.15.4 physical layer and layer / data link layer, composed of network topology, routing, security-related stack several layers.

Application layer application layer contains an application running on a network node, the node given its own function. The main function of the application layer is the input into digital data, or digital data into output. 


\section{ZigBee network topology}

In the ZigBee network, according to the equipment of the communication ability, can be divided into fully functional device FFD RFD and reduced function devices. Between FFD and FFD and RFD can communicate with each other; But RFD can only communicate with FFD, not communicate with other RFD [6]. RFD is mainly used for control application of the simple, less the amount of data transferred, the transfer and communication resources takes less, can use the scheme of realization of relatively cheap, usually as a communication terminal in the network structure. FFD requires function relatively strong MCU, generally in the network structure has the function of the network control and management. ZigBee network, there is a called PAN network coordinator (FFD equipment, it is the center of the network nodes. PAN network coordinator in addition to directly involved in the application, also responsible for the other members of the network identity management, link-state information management, and packet forwarding, and other functions [7].

From the point of view of network topology, ZigBee equipment mainly has three kinds of roles: coordinator, network routers and network terminal equipment. The network coordinator is mainly responsible for the establishment of the network, and network related configuration; Router is mainly responsible for looking for, build and repair the network packet routing information, and is responsible for forwarding the network packets; Network terminal have to join, exit network function, and can send and receive network packets, but not allow routing forwarding message terminal equipment. Usually coordinator and router nodes generally consists of FFD function equipment, terminal equipment consists of RFD equipment.

ZigBee network support star, mesh and cluster-like three network topology, as shown in Figure 2, left to right is a star network, tree networks and mesh networks.
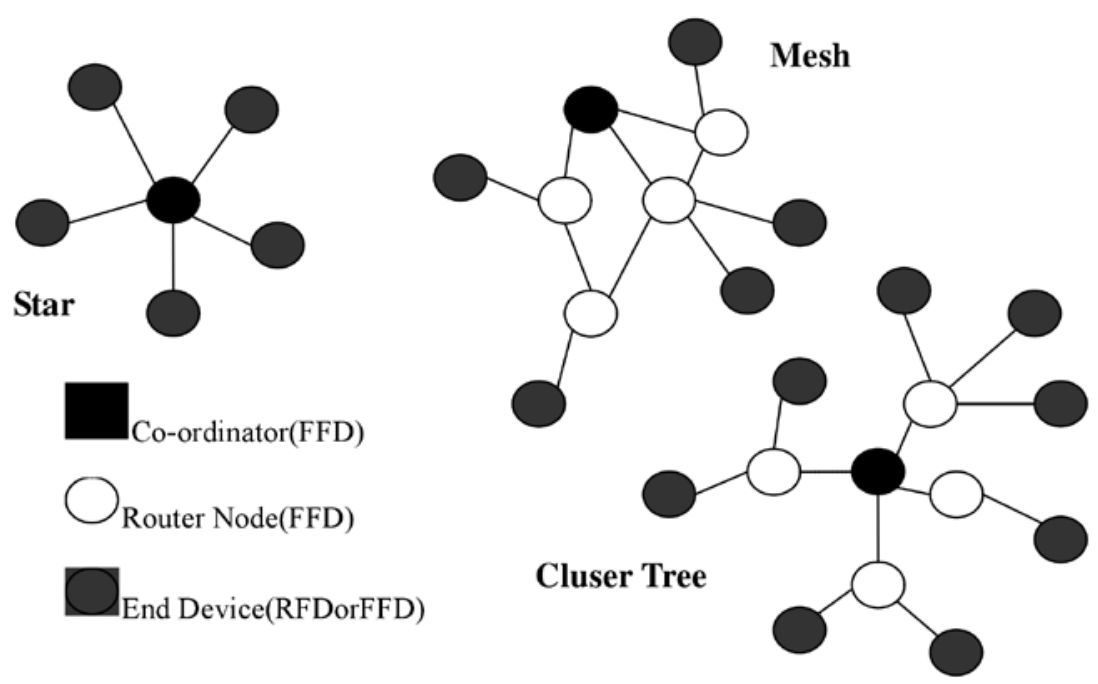

Figure2. ZigBee network topology

Star network. All equipment center equipment PAN coordinator to communicate with the network, in fact, this simple router network structure is no routing function. In this network configuration, network coordinator general electricity supply system, and other devices are battery powered. Star network for home automation, personal computer peripherals and personal health care and other small range of indoor applications.

Mesh network. Only need each other within each other's radio radiation range, between any two devices can communicate directly FFD in Mesh network each FFD device can be considered a network router, you can achieve the network packet routing and forwarding capabilities, Mesh network is more complex when building, node to maintain the information more.

Clustered network. It can actually be seen as a complex star network, an extended star topology or topology from multiple simple star network composed in clustered network, the network coordinator, is by and terminal equipment The function is clear, with respect to the Mesh 
network, build clustered network is relatively simple, relatively few resources required, and can achieve forwarding route network, thus expanding the communication range of the network.

\section{ZigBee network system design and implementation}

Network node equipment in wireless sensor networks have the effect of supervisory control and data acquisition, in star network, it is the child nodes of the network coordinator, which can be connected to the net as a compact terminals, which can reduce the amount of calculation and save the energy, power consumption is a problem of sensor network priority.

ZigBee network nodes to achieve the main function is to join an existing wireless LAN, access to sensor data, then communicate with each other and the network coordinator. Network equipment nodes also control both the information gatherers performer information. The following are application-layer network device node development step and detail, some of the key steps is omitted here similar to the coordinator.

- Active channel scan. Device sends a beacon request command of beacon frames within its working range of any domain coordinator launch lock, before the device access to the network, the device can use the active channel scan, and then select the PAN ID, the last to join selected network. During an active scan, MAC layer will discard all physical layer data services sent to non-beacon frame information.

- Active channel scan processing. Receiving from the MAC management entity to the active channel scanning acknowledgment message, the message is extracted in the descriptor list PAN, PAN descriptor includes a lot of information, such as coordinate address mode, the PAN coordinator identifier, the beacon frame address of the coordinator, the choice of logical network, link quality beacon transmission time of beacon frames received beacon frame option whether to use the security mechanism in the security process frame for errors, and so on.

- Indirect transmission. Coordinator sends data to the device is used in an indirect child node of transmission, the coordinator first stores the data together, and then wait for the device node data requests. In non-beacon networks, device nodes in order to save power, most of the time in sleep mode, periodically wake enter active mode, with a request to send data frames to the coordinator, the coordinator exist if you want to send this node devices data, it replies with a response frame data, otherwise, would reply to a response frame data field without any data.

- Upper layer data control protocol. If the child node device receives data from the coordinator of the first one byte is $0 \times 01$, then show the first two bytes of data are used to control the display of the child node status LED on the device. If the child node device receives data from the coordinator of the first one byte is $0 \times 02$, the data indicates that the child node coordinator command sends the current situation as well as LED lights display the various sensors to the coordinator.

In the ZigBee technology alliance, Freescale, Ti, Chipcon, Philips and other companies are the pioneer of ZigBee standards. In the aspect of rf transceiver chip, basically have Freescale MC13192, MC13193 and Chipcon company CC2420, CC2430 provides two solutions. In a laboratory study of Freescale series MCU has a long-term, of Freescale company 8-bit, 16-bit and 32-bit MCU series of burning, start, and the internal function modules are familiar with, based on the above consideration, provided by the paper chose the Freescale company ZigBee hardware platform: MC9S08GB60 and MC13192, its ZigBee system structure is shown in figure 3. 


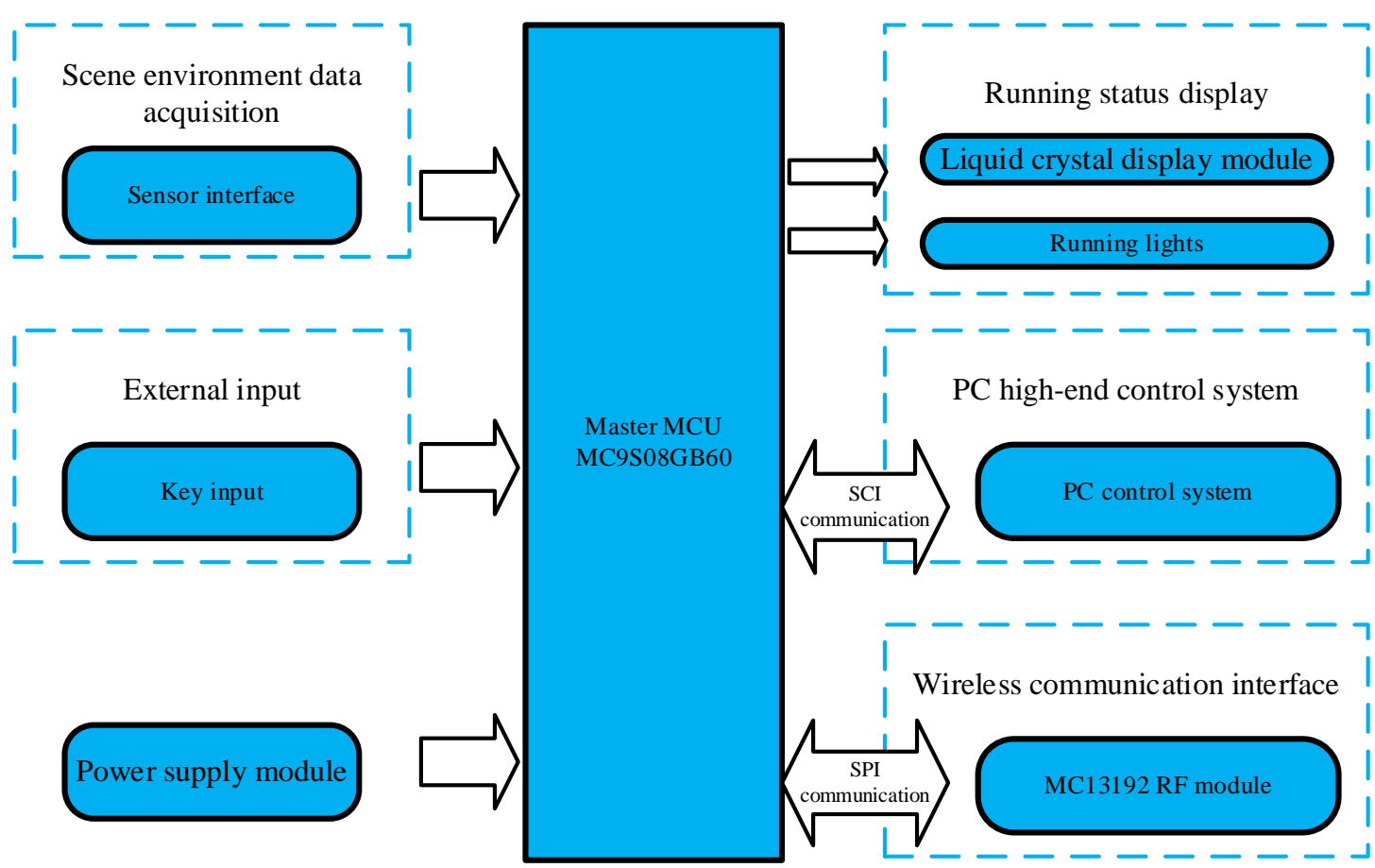

Figure 3. The stricture of ZigBee network system

Where the master MCU and its supporting module and power module is a complete hardware system, the basic part, GB60 responsible for controlling the power supply module is responsible for the overall system power supply for all modules; MC13192 RF $2.4 \mathrm{GHz}$ wireless RF module is the basis for it mainly through communication between GB60 SPI interface; operating status display including LCD and running lights, which is mainly used to display the current system status, and can also bring convenience to detect and program debugging hardware module; MT-ZigBee communication via the serial interface and control system of the PC to communicate, so that you can perform on the ZigBee network directly through the PC operating; Finally, taking into account the specific applications, hardware system also joined the field of environmental data collecting inputs and buttons, providing the appropriate interface for analog applications of agricultural greenhouses. For key input, SCI serial communication modules, LCD and running lights module design is relatively simple, not described. The following highlights the hardware design of the power supply module, MCU support module, GB60 and MC13192 and MC13192 RF interface circuit communication module.

\section{Conclusion}

The potential value of the wireless sensor network (WSN), the research of wireless sensor networks at home and abroad is in its infancy, from theory research to the engineering practice of many links need to be further perfect. ZigBee network as an emerging wireless communication technology, the network powerful, easy to use, low cost, low power consumption, application field will be more and more widely. Aiming at the requirements of the subject of some key technologies in wireless sensor networks based on ZigBee carried on the thorough research and implementation. Large-scale commercial application of ZigBee technology remains to be time, but it was already showing the special application value, believe that with the development of related technologies and propulsion, will have more applications. However, we should also clearly realize that the wireless network based on ZigBee technology has only just begun development, its technology, the applications are still far from mature, domestic enterprises should seize the opportunity, increase investment, and promote the development of the industry. 


\section{Acknowledgements}

Science and technology of Nanyang 2013GG053.

\section{References}

[1] Sung W T, Hsu Y C. Designing an industrial real-time measurement and monitoring system based on embedded system and ZigBee[J]. Expert Systems with Applications, 2011, 38(4): 4522-4529.

[2] Malhi K, Mukhopadhyay S C, Schnepper J, et al. A Zigbee-based wearable physiological parameters monitoring system[J]. Sensors Journal, IEEE, 2012, 12(3): 423-430.

[3] Han D M, Lim J H. Design and implementation of smart home energy management systems based on zigbee[J]. Consumer Electronics, IEEE Transactions on, 2010, 56(3): 1417-1425.

[4] Yiming Z, Xianglong Y, Xishan G, et al. A design of greenhouse monitoring \& control system based on ZigBee wireless sensor network[C]//Wireless Communications, Networking and Mobile Computing, 2007. WiCom 2007. International Conference on. IEEE, 2007: 2563-2567.

[5] Han D M, Lim J H. Smart home energy management system using IEEE 802.15. 4 and zigbee[J]. Consumer Electronics, IEEE Transactions on, 2010, 56(3): 1403-1410.

[6] Kim B, Kim Y, Lee I S, et al. Design and implementation of a ubiquitous ECG monitoring system using SIP and the Zigbee network[C]//Future generation communication and networking (fgcn 2007). IEEE, 2007, 2: 599-604.

[7] Gungor V C, Hancke G P. Industrial wireless sensor networks: Challenges, design principles, and technical approaches[J]. Industrial Electronics, IEEE Transactions on, 2009, 56(10): 4258-4265. 